Article

\title{
Energy and Efficiency Evaluation of Feedback Branch Design in Thermoacoustic Stirling-Like Engines
}

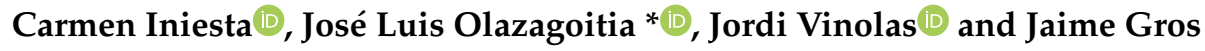 \\ Industrial Engineering and Automotive, Nebrija University, Campus de la Dehesa de la Villa, Calle Pirineos, 55, \\ 28040 Madrid, Spain; miniesta@nebrija.es (C.I.); jvinolas@nebrija.es (J.V.); jgros@nebrija.es (J.G.) \\ * Correspondence: jolazago@nebrija.es
}

Received: 20 September 2019; Accepted: 10 October 2019; Published: 12 October 2019

check for updates

\begin{abstract}
Stirling-like thermoacoustic generators are external combustion engines that provide useful acoustic power in the absence of moving parts with high reliability and respect for the environment. The study of these systems involves a great complexity since the parameters that describe them, besides being numerous, present a high degree of coupling between them. This implies a great difficulty in characterizing the effects of any parametric variation on the performance of these devices. Due to the huge amount of data to analyze, the experiments and simulations required to address the problem involve high investments in time and resources, sometimes unaffordable. This article presents, how a sensitivity analysis applying the response surface methodology can be applied to optimize the feedback branch of a thermoacoustic Stirling-like engine. The proposed study is made by evaluating the comparative relevance of seven design variables. The dimensional reduction process identifies three significant factors: the frequency of operation, the internal diameter of compliance, and the inertance. Subsequently, the Response Surface Methodology is applied to assess the interaction effects of these three design parameters on the efficiency of the thermoacoustic engine, and an improvement of $6 \%$ has been achieved. The enhanced values given by the response surface methodology are validated using the DeltaEC software.
\end{abstract}

Keywords: thermoacoustics; Stirling-like engine; efficiency; thermoacoustic engine; acoustic feedback

\section{Introduction}

It is interesting to point out that only between $12 \%$ and $30 \%$ of the energy in a vehicle fuel tank is actually transformed in energy that moves the car along the road [1] (Figure 1). Nearly $60 \%$ is lost through the exhaust pipe. For this reason, several technologies aiming at recovering this energy are actively being investigated, among others are organic Rankine cycle (ORC) [2], thermoacoustic Stirling-type cycle generators (TA-SLiCE) [3], and also thermoelectric generators (TE) [4]. Figure 2 shows a very simple sketch of the principle, where the high temperature of the exhaust gas of the combustion engine is used as the heat source of a Thermoacoustic engine. Two heat exchangers are required, the hot heat exchanger (HHX) which is connected to the exhaust gas flow and provides thermal energy to the TA-SLiCE and a cold heat exchanger (CHX). The temperature of the exhaust gasses very much depends on the engine operating conditions and could vary from $400^{\circ} \mathrm{C}$ to $650{ }^{\circ} \mathrm{C}$ at full load as indicated in [5], but still these conditions offer a good potential for energy harvesting. 


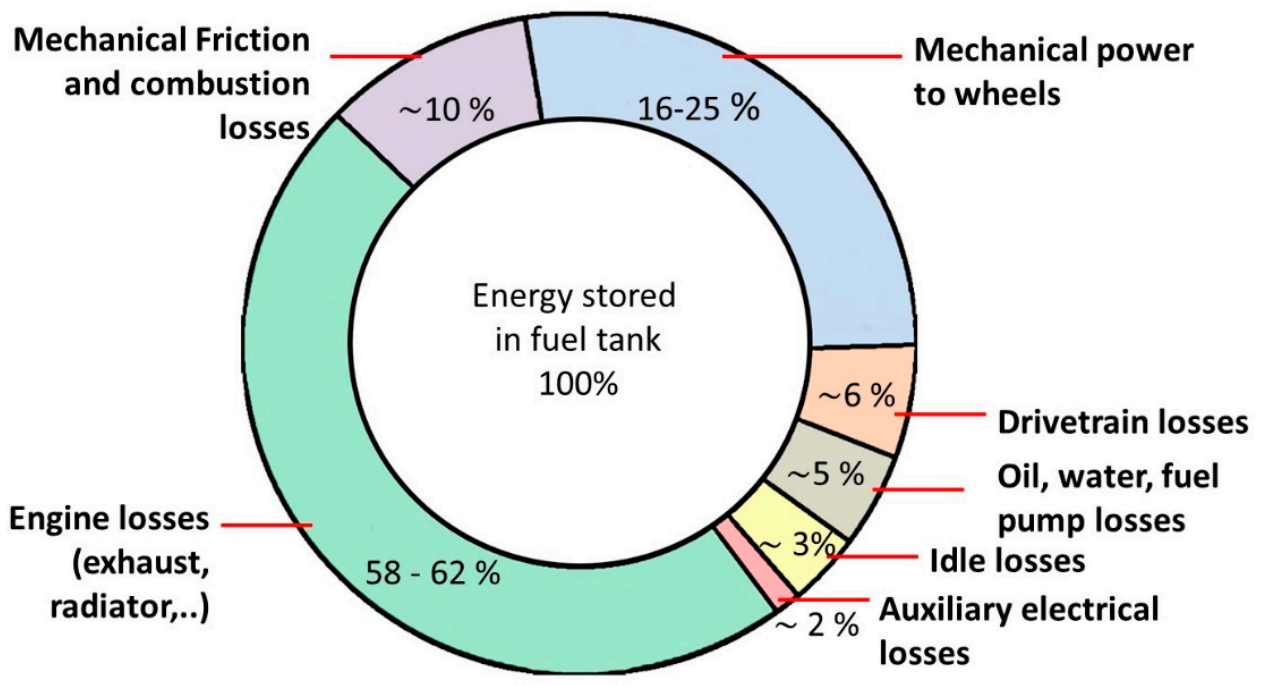

Figure 1. Energy requirements for combined city/highway driving (adapted from [1]).

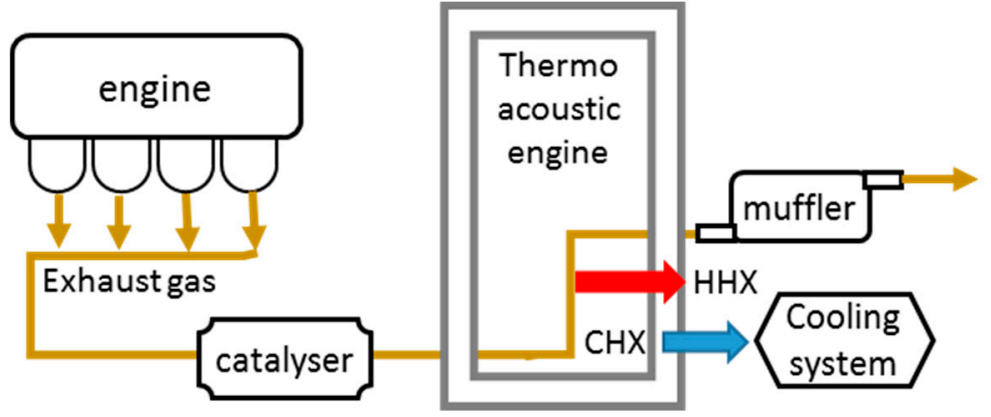

Figure 2. Sketch of the system to recover energy from the exhaust gas of a combustion engine using a loop-branched Stirling-type cycle generator (TA-SLiCE) and showing the heat exchange through the Hot and Cold Heat Exchanger (HHX and CHX respectively).

A TA-SLiCE is a good option to be part of the energy recovery system as they have a simple configuration with no moving parts, and are therefore more reliable, have low manufacturing costs, and reduced maintenance. Because they are external combustion engines, they can be powered by renewable thermal sources as well as thermal energy dissipated in other processes as is the case for the exhaust as of a combustion engine. The TA-SLICE encloses a compressible fluid suitable to sustain a sound wave. The core branch of the device contains a hot heat exchanger (HHX), a main cold heat exchanger (CHX), a regenerator (REG) interposed between the heat exchangers to maintain a temperature gradient in the compressible fluid, and a thermal buffer tube (TBT). The core branch ends after a secondary cold exchanger which is attached to a T-junction (named "Junction" in Figure 3 that splits the power flow into two additional branches: the feedback and the power extraction branches. The so-called feedback branch is a passive acoustic circuit formed by tubes, elbows, and section changes. It serves as a waveguide, redirecting the power flow at the beginning of the core branch to feed the thermoacoustic effect. After the core branch, at "Junction", the velocity is delayed with respect to the pressure. Thus, another important task of the feedback branch is to adapt the phasing between pressure and velocity corresponding to a progressive wave. The power extraction branch includes an acoustic RC-load and a mechanical resonator with an oscillating piston. 


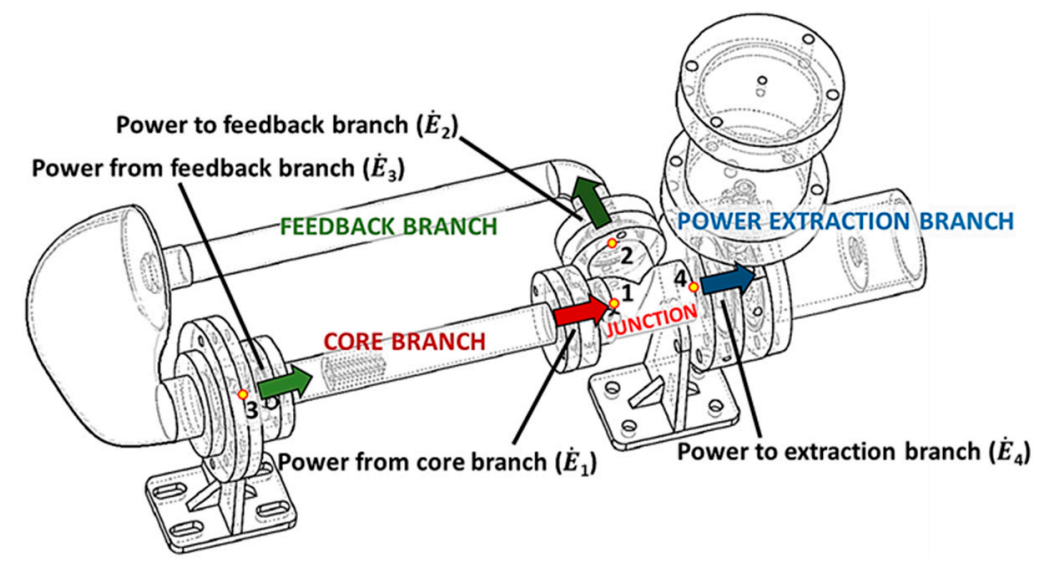

Figure 3. Schematic illustration of the distribution of acoustic power circulating through the three feedback branches in the TA-SLiCE prototype.

Despite the great achievements of the research on TA-SLiCE [6], their power density and thermoacoustic efficiency are relatively low in practice [7]. This accentuates the importance of an enhanced design of the thermoacoustic engine and this is precisely the objective of the paper: performing a sensitivity analysis of design parameters in a TA-SLiCE. In the paper, seven parameters can be varied in the design enhancement process (Compliance Inner diameter, Compliance Length, Inertance Inner diameter, Inertance Length, TBT Length, Heater Length, and Resonance Frequency). The range of variation is $\pm 25 \%$ and delimits the high and low values of each parameter

As can be deduced looking at the parameters to be varied, the focus of attention in this paper is the design improvement of the feedback branch, but the methodology exposed could be extended to other parts of the engine. The analysis of this branch, which inertance and compliance act as a phase regulator, is relevant, given that it has the function of delivering the wave to the core branch with a phase difference characteristic of a progressive wave. Due to the numerous parameters needed to describe these devices, which also have a high interaction between them [8], it is not possible to know in advance their optimal functional conditions. Therefore, in this article, a procedure for optimizing the performance of a TA-SLiCE prototype is presented, based on the relevant parameters, which had previously been screened by a statistical dimensional reduction method. The objective, when applying this procedure, is to understand the influence of these on the thermoacoustic efficiency at "Junction", under statistical control of the results.

The most widely used software to model and design a variety of complicated thermoacoustic devices, named Design Environment for Low-Amplitude Thermoacoustic Energy Conversion (DeltaEC) [9], has become a standard tool for performance analysis [10]. There are many references in which the results of TA-SLiCE models built in DeltaEC are compared to experiments and show a very good correlation [11]. Therefore it has been used by many research and industry lead projects to model, analyze, and identify potential system improvements for both efficiency and total power output [12]. The program handles a numerically 1D wave equation, the geometry and gas is defined by the user, and specific acoustic elements such as resonators, duct networks, regenerators, heat exchangers, etc. are included in order to be able to have a realistic representation of the actual system. DeltaEC has been used in other cases for the prediction and parametric analysis of thermoacosutic engines, reference [13] reports a study of acoustic streaming (Gedeon streaming) in a thermoacoustic engine with a jet pump.

Some studies, for the optimization of thermoacoustic refrigeration devices, apply the response surface methodology (RSM) [14] and, more recently, the Taguchi method [15]. Other works have also applied RSM for the optimization of standing-wave thermoacoustic engines, mainly focused on considering stack [16] and resonator [17] design parameters. Nevertheless, few studies including a sensitivity analysis and RSM optimization of TA-SLiCE have been reported to date. Such investigations are focused on the analysis of acoustic streaming and jet pump optimization [18] in a thermoacoustic 
Stirling heat engine. This is representative of the great complexity involved in the study of TA-SLiCE and the great difficulty involved in characterizing the effects of any system variation on its final function. Due to the extensive amount of data to be analyzed, the experiments and simulations required to establish a complete parametric configuration entail a high investment in time and resources, sometimes unacceptable through a simple analysis of the effect of parameter variation.

The paper is organized as follows: Section 2 presents the base DeltaEC model of the TA-SLiCE prototype chosen to be part of the design enhancement process, Section 3 describes how the factorial Design Of Experiments methodology will be used and which of the TA-Slice parameters will be varied, Section 4 provides detail on the virtual results supported by the sensitivity analysis and RSM, and Section 5 defines the most relevant conclusions and open points for future studies.

\section{DeltaEC Model of the TA-SLiCE Prototype}

According to the linear thermoacoustic theory applied by DeltaEC, the mean acoustic power flux circulating in the $x$-direction through the one-dimensional passive acoustic circuit configured by segments is given by the following second order expression, according to Rott's approximation [8]:

$$
\dot{E}(x)=\frac{1}{2}\left|p_{1}\right|\left|U_{1}\right| \cos \varnothing_{p U}
$$

where $\varnothing_{p U}$ is the angle between the pressure $p_{1}$ and the velocity $U_{1}$ of the acoustic wave. The acoustic power to be achieved largely depends on this angle, since the flux through the passive acoustic circuit of TA-SLiCE is determined solely by the characteristics of the connected load. The total acoustic impedance of an acoustic circuit $\left(Z_{a}\right)$ is the sum of all its constituent elements (or segments) [8]:

$$
Z_{a}=p / U=R_{a}+j\left(\omega L_{a}-1 / \omega C_{a}\right)
$$

Thus, $Z_{a}$ defines the resistive, inductive, and capacitive segments of which acoustic circuits are made. These elements are the acoustic resistance $R_{a}$, the inertance $L_{a}$, and the compliance $C_{a}$. The impedance of these segments influences the pressure $\left|p_{1}\right|$ and velocity $\left|U_{1}\right|$, and consequently impacts on the behavior of the wave propagation. Therefore, the feedback branch of a TA-SLiCE plays a decisive role in tuning $\varnothing_{p U}$ so that the acoustic wave reaches the regenerator in the best progressive wave conditions. In this case, the thermodynamic cycle (micro-scale Stirling-like) takes place in its most efficient way.

The analysis is carried out using the TA-SLICE shown in Figure 3. The geometric and operational parameters for each required segment to model it in DeltaEC are shown in Table 1. The BEGIN segment in Table 1 sets the operating conditions with the linear thermoacoustic theory by setting the ratio between the pressure amplitude and the mean pressure at $5 \%$. The active core is inside the TBT, therefore all the components of the core branch have the same cross section of $0.00025 \mathrm{~m}^{2}$, as does the TBT. The feedback branch is modeled by arranging two tubes (DUCT segments) in series, one acting as an inertance and the other as a compliance. The mechanical resonator has been modeled using an IESPEAKER segment as a piston and a closed volume behind it, which acts as a gas spring. 
Table 1. Geometry details and operating conditions of the proposed TA-SLiCE model. Design Environment for Low-Amplitude Thermoacoustic Energy Conversion, DeltaEC.

\begin{tabular}{|c|c|}
\hline DeltaEC Segment Configuration & Virtual Value \\
\hline \multicolumn{2}{|c|}{ BEGIN Operating Conditions } \\
\hline Working gas & Air \\
\hline Mean pressure $\left(p_{m}\right)$ & $100 \mathrm{kPa}$ \\
\hline Resonance frequency $(f)$ & $40 \mathrm{~Hz}$ \\
\hline Initial temperature of the gas $\left(T_{m}\right)$ & $328 \mathrm{~K}$ \\
\hline Initial value of pressure amplitude $\left(\left|p_{1}\right|\right)$ & $5 \mathrm{kPa}$ \\
\hline \multicolumn{2}{|l|}{ Core Branch } \\
\hline \multicolumn{2}{|c|}{ Main Cooler (CHX) } \\
\hline Length $\left(L_{C H X}\right)$ & $0.014 \mathrm{~m}$ \\
\hline Porosity $\left(\phi_{C H X}\right)$ & $60 \%$ \\
\hline Heat output $\left(\dot{Q}_{C 1}\right)$ & $6 \mathrm{~W}$ \\
\hline \multicolumn{2}{|c|}{ Regenerator (REG) } \\
\hline Length $\left(L_{R E G}\right)$ & $0.013 \mathrm{~m}$ \\
\hline Porosity $\left(\phi_{R E G}\right)$ & $92 \%$ \\
\hline Hydraulic radius $\left(r_{h R E G}\right)$ & $0.0002 \mathrm{~m}$ \\
\hline \multicolumn{2}{|c|}{ Heater (HHX) } \\
\hline Length $\left(L_{H H X}\right)$ & $0.029 \mathrm{~m}$ \\
\hline Porosity $\left(\phi_{H H X}\right)$ & $60 \%$ \\
\hline Heat input $\left(\dot{Q}_{H}\right)$ & $8 \mathrm{~W}$ \\
\hline \multicolumn{2}{|c|}{ Thermal buffer tube (TBT) } \\
\hline Length $\left(L_{T B T}\right)$ & $0.101 \mathrm{~m}$ \\
\hline Inner diameter $\left(D_{T B T}\right)$ & $0.018 \mathrm{~m}$ \\
\hline \multicolumn{2}{|c|}{ Secondary Cooler (SCHX) } \\
\hline Length $\left(L_{S C H X}\right)$ & $0.01 \mathrm{~m}$ \\
\hline Porosity $\left(\phi_{S C H X}\right)$ & $70 \%$ \\
\hline Heat output $\left(\dot{Q}_{\mathrm{C} 2}\right)$ & $1.9 \mathrm{~W}$ \\
\hline \multirow{2}{*}{\multicolumn{2}{|c|}{$\begin{array}{l}\text { Feedback Branch } \\
\text { Compliance (DUCT) }\end{array}$}} \\
\hline & \\
\hline Inner diameter $\left(D_{C}\right)$ & $0.0435 \mathrm{~m}$ \\
\hline Length $\left(L_{C}\right)$ & $0.126 \mathrm{~m}$ \\
\hline \multicolumn{2}{|c|}{ Inertance (DUCT) } \\
\hline Inner diameter $\left(D_{L}\right)$ & $0.016 \mathrm{~m}$ \\
\hline Length $\left(L_{L}\right)$ & $0.187 \mathrm{~m}$ \\
\hline \multicolumn{2}{|c|}{$\begin{array}{l}\text { Power Extraction Branch } \\
\text { Mechanical Resonator (IESPEAKER) }\end{array}$} \\
\hline Length $\left(L_{R E S}\right)$ & $0.145 \mathrm{~m}$ \\
\hline Piston area $\left(A_{R E S}\right)$ & $0.0013 \mathrm{~m}^{2}$ \\
\hline Piston weight $\left(M_{R E S}\right)$ & $0.01 \mathrm{~kg}$ \\
\hline Inner diameter $\left(D_{R E S}\right)$ & $0.04 \mathrm{~m}$ \\
\hline Mechanical resistance $\left(R_{m}\right)$ & $0.06 \mathrm{~N} \cdot \mathrm{s} \mathrm{m}^{-1}$ \\
\hline
\end{tabular}

The virtual results at various locations of the prototype numbered in Figure 3 are disclosed in Table 2 which shows the virtual results of the rise $\Delta E_{\text {core }}=\dot{E}_{1}-\dot{E}_{3}$, and drop $\left|\Delta E_{f e e d b a c k}\right|=\dot{E}_{3}-\dot{E}_{2}$, of acoustic power produced through the core and feedback branches, respectively. 
Table 2. Performance results at different locations of the proposed TA-SLiCE model.

\begin{tabular}{cc}
\hline Location \& Measurement & Virtual Result \\
\hline (1) Acoustic power from core branch $\left(\dot{E}_{1}\right)$ & $1.350 \mathrm{~W}$ \\
(2) Acoustic power to feedback branch $\left(\dot{E}_{2}\right)$ & $0.855 \mathrm{~W}$ \\
(3) Acoustic power from feedback branch/to core branch $\left(\dot{E}_{3}\right)$ & $0.782 \mathrm{~W}$ \\
(4) Acoustic power to power extraction branch $\left(\dot{E}_{4}\right)$ & $0.496 \mathrm{~W}$ \\
Acoustic power at RC load $\left(\dot{E}_{\mathrm{RC}}\right)$ & $0.238 \mathrm{~W}$ \\
Acoustic power rise through core branch $\left(\Delta E_{\text {core }}\right)$ & $0.568 \mathrm{~W}$ \\
Acoustic power drop through feedback branch $\left(\Delta E_{\text {feedback }}\right)$ & $0.073 \mathrm{~W}$ \\
Thermoacoustic efficiency $\left(\eta_{t a}\right)$ & $6.199 \%$ \\
\hline
\end{tabular}

In order to qualitatively study the overall performance of the TA-SLiCE it is necessary to define the thermoacoustic efficiency of the system that is given by:

$$
\eta_{t a}=\frac{\dot{E}_{4}}{\dot{Q}_{H}}
$$

where $\dot{Q}_{H}$ is the external thermal power supplied. As can be appreciated by both Figure 3 and Table 2 , the acoustic power $\dot{E}_{4}$ which is not derived to the feedback branch, is a surplus power (usable acoustic power) released to the power extraction branch:

$$
\dot{E}_{4}=\dot{E}_{1}-\dot{E}_{2}
$$

According to DeltaEC results, the TA-SLiCE of Figure 3 reaches an efficiency of approximately $6.2 \%$. The precision of the virtual results obtained with DeltaEC in this document is given by the tolerance of the convergence parameter that, in all cases, satisfies a value lower than $10^{-8}$. In addition, given the $5 \%$ transmission ratio that is inferred from the initial conditions given in the BEGIN segment, the assumption is that the engine operates in conditions of low "drive ratio" $(<10 \%)$. In these circumstances, the precision of the virtual results with DeltaEC are universally accepted and, therefore, the presented model is reliable for the present study.

Looking at the value of the efficiency, $6.2 \%$ in this case, one could think that it is low and even question the value of such an energy harvesting system. However, two points have to be kept in mind that favor such systems. Firstly, it is recovering from an energy that would otherwise be wasted, in this case through the exhaust of the combustion engine. Secondly recovering such energy in a combustion engine would directly imply a direct reduction in fuel consumption and any step in that direction is valuable.

The objective of the paper is not to carry out a theoretical-experimental investigation of the TA-SLiCE, however some tests have been carried out using a prototype built as indicated in Figure 4 and Table 1. The aim is to verify the DeltaEC model that will be used in the following sections at least with some experimental values. In this case it was possible to measure the acoustic power at RC load $\left(\dot{E}_{\mathrm{RC}}\right)$ with three different configurations varying the inertance and compliance diameter and length (Figure 4). There is a good trend agreement between experiments and the results provided by the DeltaEC model. The better performance predicted by DeltaEC is due to friction of the mechanical resonator in the prototype. In fact, it is not possible, even with very precise tolerances when manufacturing the resonator, to have both low friction and at the same time keep the acoustic circuit sealed. Overall the modeling results predict the thermoacoustic performance satisfactorily. As a general consideration when the ratio of pressure amplitude to the mean pressure is less than $10 \%$, the nonlinear effects in the TA-SLiCE are negligible, and DeltaEC can be used to predict and analyze its performance as the linear thermoacoustic theory prevails. 


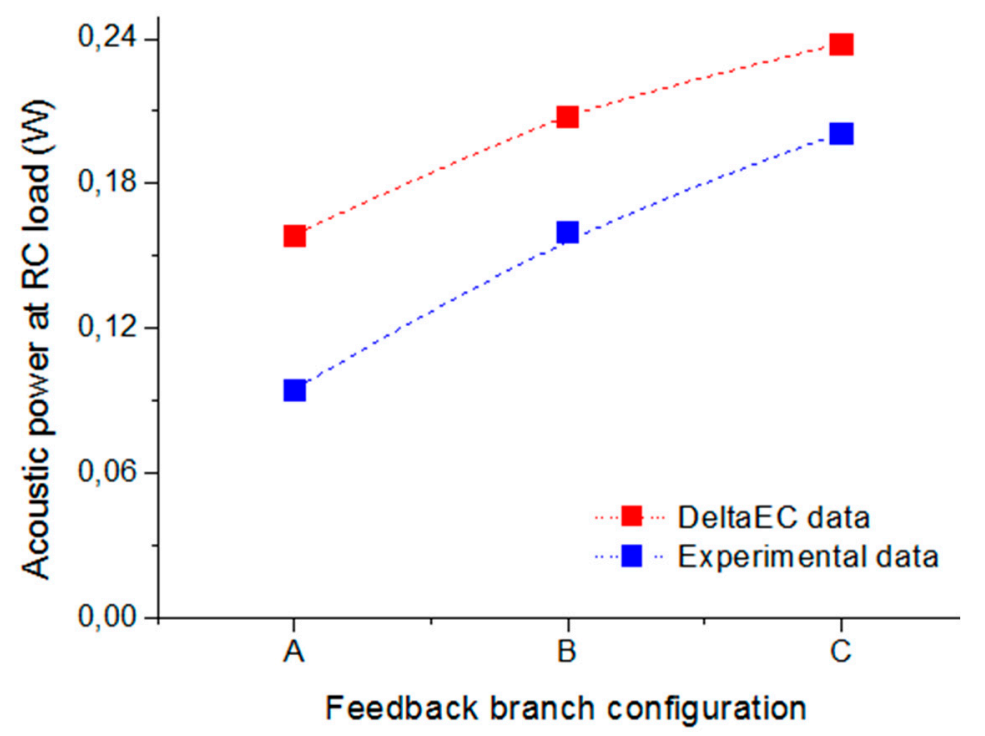

Figure 4. Comparison of the thermoacoustic performance by DeltaEC and experimental data. Feedback branch compliance increases between case $A$ and $C$, while the length is reduced. $C$ is defined in Table $1\left(D_{C}=0.435 \mathrm{~m}, L_{C}=0.187 \mathrm{~m}, D_{L}=0.016\right.$, and $\left.L_{L}=0.187\right)$. Case A corresponds to $\left(D_{C}=0.02 \mathrm{~m}\right.$, $L_{C}=0.41 \mathrm{~m}, D_{L}=0.015$, and $\left.L_{L}=0.185\right)$. Case B corresponds to $\left(D_{C}=0.025 \mathrm{~m}, L_{C}=0.25 \mathrm{~m}, D_{L}=0.015\right.$, and $\left.L_{L}=0.24\right)$.

\section{Methodology for Design Improvement}

Starting from the results of the previous section, the design of the system is enhanced by optimizing some of the prototype parameters. Factorial designs are widely used in experiments involving several factors where it is necessary to study the combined effect of the factors on the system response [19]. As shown below, a fractional factorial design (FFD) is written and managed using the RStudio software [20] to establish an appropriate sensitive analysis for the RSM application success.

\subsection{Factors and Response Variable Selection}

The thermoacoustic efficiency $\eta_{t a}$ entails a trade-off between usable acoustic power $\dot{E}_{4}$, and power that is spent in the system feedback $\dot{E}_{2}$. It is therefore a good indicator of the performance of the device and, in the present work, used to study the response to the variation of the studied factors. The impact of these factors on the response variable will be measured and analyzed, in order to maximize the thermoacoustic efficiency.

In this study, the seven parameters of Table 3 have been selected to describe the configuration of TA-SLiCE. The range for each of the factors is delimited by the high and low levels. It is usually accepted [21] that the range of analysis to study the influence of factors on the response is reasonably established to $25 \%$ of the extreme range. The extreme range introduces constructive restrictions in the model, since TA-SLiCE design values are considered to set its boundaries, according to experiments carried out in the lab.

Table 3. Factors and levels for $2^{7}$ factorial analysis.

\begin{tabular}{lcc}
\hline \multicolumn{1}{c}{ Factor } & Low Level (-1) & High Level (+1) \\
\hline Compliance Inner diameter $\left(D_{C}\right)$ & $39 \mathrm{~mm}$ & $48 \mathrm{~mm}$ \\
Compliance Length $\left(L_{C}\right)$ & $0.121 \mathrm{~m}$ & $0.131 \mathrm{~m}$ \\
Inertance Inner diameter $\left(D_{L}\right)$ & $13 \mathrm{~mm}$ & $19 \mathrm{~mm}$ \\
Inertance Length $\left(L_{L}\right)$ & $0.182 \mathrm{~m}$ & $0.192 \mathrm{~m}$ \\
TBT Length $\left(L_{T B T}\right)$ & $0.09 \mathrm{~m}$ & $0.112 \mathrm{~m}$ \\
Heater Length $\left(L_{H H X}\right)$ & $0.026 \mathrm{~m}$ & $0.032 \mathrm{~m}$ \\
Resonance Frequency $(f)$ & $37 \mathrm{~Hz}$ & $43 \mathrm{~Hz}$ \\
\hline
\end{tabular}


If a full factorial design, with all the combinations of each factor in the two levels proposed, was to be carried out, $2^{7}=128$ experiments or simulations would be required. Therefore, a factorial design to reduce the high amount of simulations would be very beneficial.

\subsection{Dimensional Reduction $\left(2_{I V}^{7-3}\right.$ FFD)}

In order to identify the number of significant factors, an FFD is made. From now on, for greater simplicity, letters will be assigned to the factors as shown in Table 4. An RStudio script has been developed, which detects the significant factors, adjusting the linear model to the response values. The $\mathrm{R}$ code used also allows the visualization of the results by contour and Pareto plots.

Table 4. Reduced $2_{I V}^{7-3}$ fractional factorial design (FFD) for seven factors and 16 experiments.

\begin{tabular}{ccccccccc}
\hline Simulation & A & B & C & D & E & F & G & Thermoacoustic Efficiency $y=\eta_{t a}[\%]$ \\
\hline 1 & -1 & -1 & -1 & -1 & -1 & -1 & -1 & 6.0072 \\
2 & +1 & -1 & -1 & -1 & +1 & +1 & +1 & 6.2118 \\
3 & -1 & +1 & -1 & -1 & +1 & +1 & -1 & 6.4742 \\
4 & +1 & +1 & -1 & -1 & -1 & -1 & +1 & 6.3441 \\
5 & -1 & -1 & +1 & -1 & +1 & -1 & +1 & 4.5394 \\
6 & +1 & -1 & +1 & -1 & -1 & +1 & -1 & 4.9740 \\
7 & -1 & +1 & +1 & -1 & -1 & +1 & +1 & 5.3908 \\
8 & +1 & +1 & +1 & -1 & +1 & -1 & -1 & 6.3343 \\
9 & -1 & -1 & -1 & +1 & -1 & +1 & +1 & 5.9103 \\
10 & +1 & -1 & -1 & +1 & +1 & -1 & -1 & 6.3628 \\
11 & -1 & +1 & -1 & +1 & +1 & -1 & +1 & 6.6115 \\
12 & +1 & +1 & -1 & +1 & -1 & +1 & -1 & 6.0218 \\
13 & -1 & -1 & +1 & +1 & +1 & +1 & -1 & 4.7358 \\
14 & +1 & -1 & +1 & +1 & -1 & -1 & +1 & 5.0878 \\
15 & -1 & +1 & +1 & +1 & -1 & -1 & -1 & 5.7468 \\
16 & +1 & +1 & +1 & +1 & +1 & +1 & +1 & 6.2608 \\
\hline
\end{tabular}

This highly fractional factorial design $2_{\mathrm{IV}}^{7-3}$ needs to run $2^{4}=16$ simulations to predict the effects of one factor in the levels of the other factors and to obtain conclusions that are valid in the range of -1 to +1 . The code " +1 " represents the highest level and " -1 " the lowest level corresponding to each factor. This design of resolution IV provides solid and robust predictions, since the confusion of the main effects will only occur with interactions of three factors and above, of little significance [22].

To obtain the confusion pattern proposed in Table 4, 16 numerical simulations have been performed in DeltaEC. The first four columns contain a complete factorial design for four factors A, B, C, and D with all the combinations of each factor in orthogonal code. The next three columns correspond to the factors $\mathrm{E}, \mathrm{F}$, and $\mathrm{G}$. They are generated by assigning values to those factors using the relations $\mathrm{E}=\mathrm{ABC}$, $\mathrm{F}=\mathrm{ABD}$, and $\mathrm{G}=\mathrm{ACD}$. The response variables of the last column $y=\eta_{t a}$ are calculated by combining the high and low levels of the factors in real notation during DeltaEC simulations.

The Pareto diagrams in Figure 5, derived from the generated FFD, show the main effects, as well as the interaction effects and their direction (positive or negative impact).

All the Pareto plots in Figure 5 visually indicate the significance of factors B and C; C has a negative effect in the response whereas $B$ has a positive one. Factors $A$ and $E$ have little significance, and factors F, D, and G are negligible coefficients. Usually model terms are selected or rejected based on the significant probability value (p-value within 5\% significance level) [22]. Factors F, D, and G are not significant, and their variation can be ignored for the response because their $\mathrm{p}$-values are greater than 0.05. In plots Figure 5b,d factors F, D, and G are no longer considered. In Figure $5 b$, the interactions of the factors are included, while in Figure $5 \mathrm{~d}$ only the main effects are visualized. 

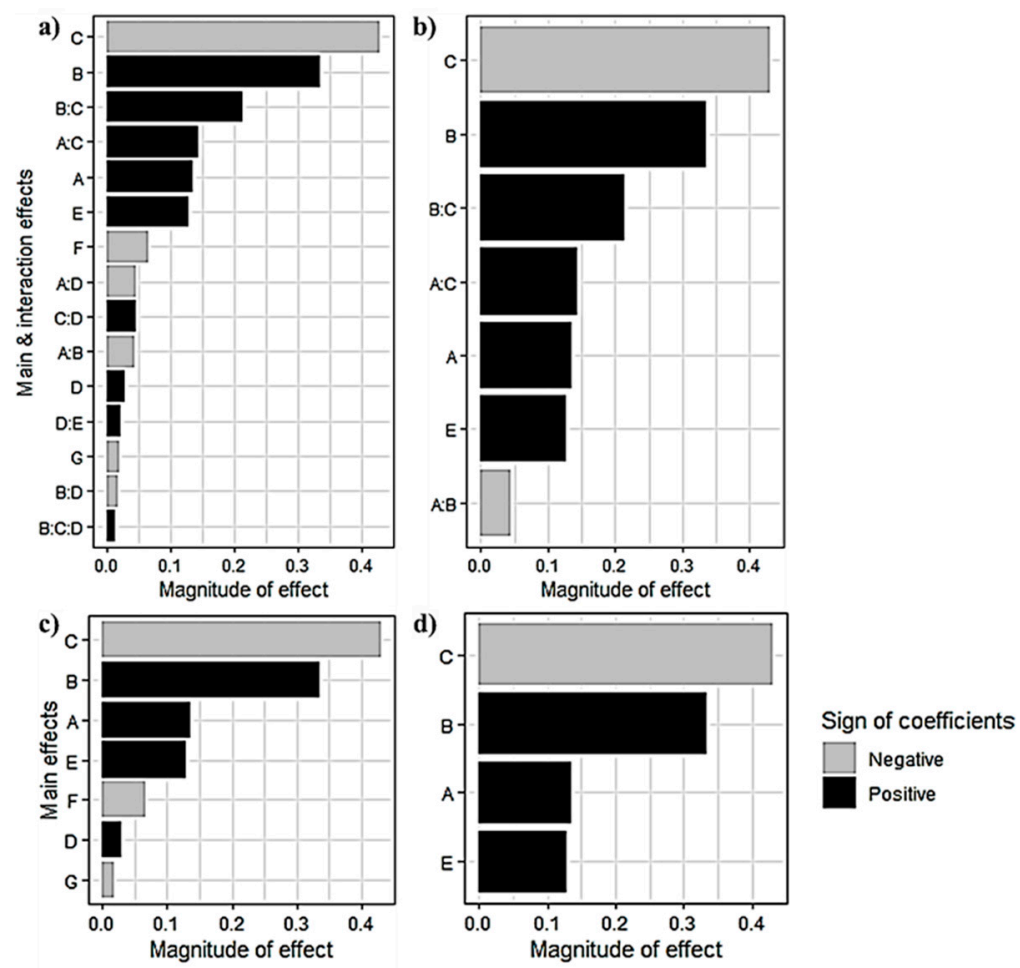

Figure 5. Pareto plots for thermoacoustic efficiency FFD of resolution IV. (a) shows the main and interaction effect: for all factors; (b) shows the same but removing the negligible F, D and G factors; (c) only the main factor effect display for all factors; and (d) the same, but removing the negligible F, D, and $G$ factors.

In order to make the model sufficiently precise and robust, it must include the interactions. Therefore, the relevance of factors $\mathrm{A}$ and $\mathrm{E}$ is set, based on the interpretation of the Pareto diagram Figure $5 b$. Of all the possible and numerous interactions between two factors, diagram Figure $5 b$ identifies the $\mathrm{BC}, \mathrm{AC}$, and $\mathrm{AB}$ interactions, thus indicating the importance of these three interactions over all other. From the results it is clearly inferred that the $B C$ and $A C$ interactions are significant. This fact indicates that factor B modifies the effect of factor C, and, although to a lesser extent, factor $A$ modifies the effect of factor $C$. This dependence implies that the factor $A$ of the model cannot be discarded although the graphs show that it has narrow relevance. However, the $\mathrm{E}$ factor and the $\mathrm{AB}$ interaction can be definitely neglected in the model.

The impact of the parameters on the thermoacoustic efficiency of TA-SLiCE, in the range determined by the limits -1 and +1 for each factor, can be summarized in the following concluding remarks:

- $\quad$ The compliance inner diameter $\left(B=D_{C}\right)$ and inertance inner diameter $\left(C=D_{L}\right)$ are critical parameters for thermoacoustic efficiency. The inertance inner diameter has a reducing effect on the thermoacoustic efficiency, while the compliance inner diameter has an increasing effect;

- The inner diameter of inertance and compliance parameters have significant and positive interaction effects $(\mathrm{BC})$;

- The operating frequency $(\mathrm{A}=f)$ has a positive and relatively smaller impact than factors $\mathrm{B}$ and $\mathrm{C}$ on thermoacoustic efficiency;

- The operating frequency and inertance inner diameter parameters have positive and mild interaction effects (AC);

- The lengths of the inertance $\left(D=L_{L}\right)$, the compliance $\left(E=L_{C}\right)$, the thermal buffer tube $(G=G=$ $\left.L_{T B T}\right)$, and the hot heat exchanger $\left(\mathrm{F}=L_{H H X}\right)$ have a negligible effect on thermoacoustic efficiency;

- The operating frequency and compliance inner diameter parameters have negligible interaction effects (AB). Similarly, all the other interactions are negligible. 
Contour plots measure the differences between the effects of one factor on the response, at different levels of another. To interpret the effects of interaction between two factors derived from the generated FFD, the contour plots, shown in Figure 6 are used.

a) Weak interaction

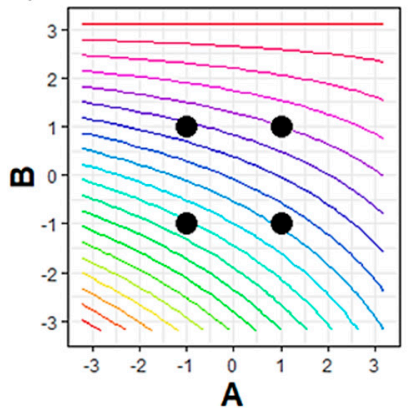

b) Medium interaction
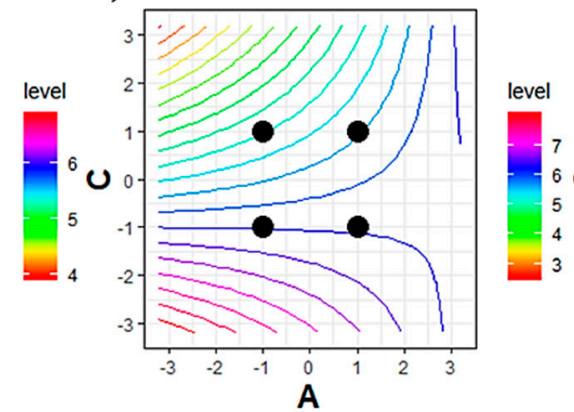

c) Strong interaction

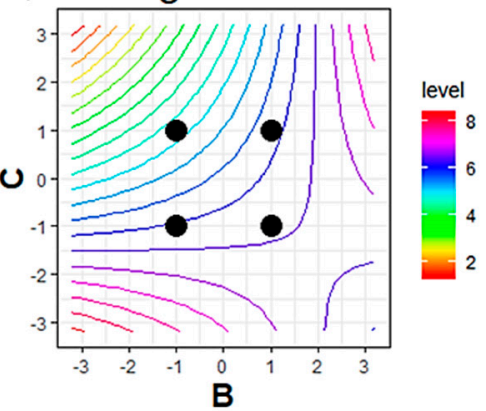

Figure 6. Contour plot for thermoacoustic efficiency. The four black points represent the high and low limits established for each factor, named "Box plot". Low (a), medium (b), and strong (c) interdependencies are detected for $\mathrm{AB}, \mathrm{AC}$, and $\mathrm{BC}$ interactions, respectively.

In Figure 6, the trend and relative magnitude of the interaction effects on thermoacoustic efficiency are visualized. The contour lines of those plots show values of the response of the model according to the level (color scale), while in the ordinate and abscissa, the levels of the two-factor interaction are shown. The four points delimit the range at levels -1 and +1 for each factor in what is called a cube plot. Plot Figure 6a shows contour lines practically parallel within the cube plot, which is interpreted as a weak interaction between factors A and B. If the lines lose the parallelism to a greater or lesser degree, the curvature of the contour lines is interpreted as evidence of interaction between the factors, so that the greater the curvature, the greater the degree of interaction. According to this reasoning, the contour plot Figure $6 \mathrm{~b}$ shows a medium interaction between factors $A$ and $C$, and an interaction of factors $B$ and $C$ somewhat stronger in the plot Figure $6 c$. This information indicates that factor B modifies the effect of factor C. Similarly, factor A modifies the effect of factor C. For the rest of interactions, where $A B$ is included, no evidence of interaction has been found.

The plots displayed above are the upshot of the least squares model derived from the $2_{I V}^{7-3}$ FFD, in terms of the relevant input parameters for thermoacoustic efficiency:

$$
\hat{y}=5.81+0.14 x_{A}+0.33 x_{B}-0.43 x_{C}+0.14 x_{A} x_{C}+0.21 x_{B} x_{C}
$$

The quality verification of the regression model in Equation (5) sets $R^{2}=0.9743$, which means that the model can explain about $97.43 \%$ of the variance in the response. Therefore, the model is acceptable for testing of statistical significance.

In the next section, the effects of the three significant factors $(A, B, C)$ and interactions $(A C, B C)$ will be further evaluated by RSM. The factors and interactions that have insignificant effects on the response will thus not be considered in what follows. D, E, F, and G factors will be established at their midpoint value " 0 " and will not vary for further simulations.

\section{Results}

Based on the sensitive analysis and dimensional reduction, the most relevant parameters are further discussed in the following paragraphs. RSM will be applied to determine the trends of the significant factors and to guide for detection of a possible local optimum within the range determined by the screening design given in Section 3. 


\subsection{Response Surface Methodology (RSM)_Faced Centered Design (FCD)}

After the screening design, the resulting significant factors are the operating frequency and the inner diameters of the inertance and of the compliance. The objective of the RMS is to increase the thermoacoustic efficiency at "Junction" to obtain the higher output of useful acoustic power from the engine. The most significant factors are the inner diameters of inertance (factor $\mathrm{C}$ ) and compliance (factor B), as well as their interaction (AB). The negative sign of factor $C$ impacts on the response, according to the predictions of the previous section. It implies that the diameter must be reduced to increase the thermoacoustic efficiency, as the objective requires. In addition, effects of factors A and B, and also interactions $\mathrm{AC}$ and $\mathrm{BC}$ have a positive impact. This result agrees with the underlying physics that occur in the feedback branch - the acoustic power losses in the inertance must be minimized with an adequate thin duct design, on the one hand, and the compliance design to minimize acoustic power losses requires being a volume with high section rather than high length, on the other. The response surface methodology combines all these effects with statistical techniques and simulated DeltaEC responses. It thus generates a high-precision prediction mathematical model. This model is fitted to a response surface, which provides a prediction of the thermoacoustic efficiency in "Junction" within the previously stablished analysis range. It therefore supplies a powerful tool for the feedback branch parametric study.

This study presents the application of a Faced centered design (FCD) with three factors (A, B, C) and three levels $(-1,0,+1)$ to generate the quadratic model. This type of RSM model is used since the system has constructive restrictions and it is not possible to leave the factorial space.

The experimental design matrix resulting from the FCD model generated in this study is shown in Table 5. The first column indicates the number of simulations carried out in DeltaEC and listed in standard order. The following six columns present the factors in "real" units and in coded units. The next two columns show the DeltaEC simulated response of thermoacoustic efficiency at "Junction" and the RSM predicted one, respectively.

Table 5. Experimental design matrix and FCD-RSM results for thermoacoustic efficiency at "Junction" for TA-SLiCE.

\begin{tabular}{|c|c|c|c|c|c|c|c|c|}
\hline Simulation & $\mathbf{A}$ & B & $\mathrm{C}$ & $\mathrm{x}_{\mathrm{A}}$ & $\mathbf{x}_{\mathbf{B}}$ & $\mathbf{x}_{\mathbf{C}}$ & $\begin{array}{c}\text { TA-Efficiency } \\
y=\eta_{t a}[\%](\text { DeltaEC })\end{array}$ & $\begin{array}{c}\text { TA-Efficiency } \\
\hat{y}=\eta_{t a}[\%] \text { (Predicted) }\end{array}$ \\
\hline 0 & 40 & 43.5 & 16 & 0 & 0 & 0 & 6.199 & 6.204 \\
\hline 1 & 37 & 39 & 13 & -1 & -1 & -1 & 6.061 & 6.029 \\
\hline 2 & 43 & 39 & 13 & +1 & -1 & -1 & 6.274 & 6.284 \\
\hline 3 & 37 & 48 & 13 & -1 & +1 & -1 & 6.592 & 6.616 \\
\hline 4 & 43 & 48 & 13 & +1 & +1 & -1 & 6.069 & 6.137 \\
\hline 5 & 37 & 39 & 19 & -1 & -1 & +1 & 4.534 & 4.469 \\
\hline 6 & 43 & 39 & 19 & +1 & -1 & +1 & 5.158 & 5.136 \\
\hline 7 & 37 & 48 & 19 & -1 & +1 & +1 & 5.693 & 5.685 \\
\hline 8 & 43 & 48 & 19 & +1 & +1 & +1 & 6.235 & 6.269 \\
\hline $\mathrm{FC}+$ & 40 & 43.5 & 19 & 0 & 0 & +1 & 5.486 & 5.546 \\
\hline FB- & 40 & 39 & 16 & 0 & -1 & 0 & 5.641 & 5.749 \\
\hline $\mathrm{FA}+$ & 43 & 43.5 & 16 & +1 & 0 & 0 & 6.372 & 6.281 \\
\hline FC- & 40 & 43.5 & 13 & 0 & 0 & -1 & 6.494 & 6.423 \\
\hline $\mathrm{FB}+$ & 40 & 48 & 16 & 0 & +1 & 0 & 6.566 & 6.447 \\
\hline FA- & 37 & 43.5 & 16 & -1 & 0 & 0 & 5.944 & 6.024 \\
\hline
\end{tabular}

The first run " 0 " located in the central point has all the factors set at the midpoint value. The next eight simulations correspond to the points located in the corners, while the last six simulations correspond to the points centered on the faces of the "cube plot". 
The DeltaEC simulated responses $y=\eta_{t a}$ are the input to generate the quadratic FCD model in terms of the relevant factors and interactions. The RSM predicted responses values $\hat{y}=\eta_{t a}$ are calculated from such model:

$$
\begin{gathered}
\hat{y}=6.204+0.128 x_{A}+0.349 x_{B}-0.438 x_{C}-0.051 x_{A}^{2}-0.106 x_{B}^{2}-0.219 x_{C}^{2}-0.102 x_{A} x_{B} \\
+0.184 x_{A} x_{C}+0.239 x_{B} x_{C}
\end{gathered}
$$

\subsection{Model Statistics}

In this section, an analysis of variance (ANOVA) was performed to verify the predictive capacity of the generated FCD model in Equation (6). The statistical results of the model for thermoacoustic efficiency at "Junction" are shown in Table 6. An F-value of 26 can be observed in Table 6 for the FCD model, which implies that the factors considered in the model have a significant effect on the response. The p-value of the model is less than 0.05 , which indicates the statistical relevance of the model at a $95 \%$ probability. In addition, Table 6 shows the main and interaction significant effects (whose p-value $<0.05)$. The effects whose $\mathrm{p}$-value is above that threshold have not been included in the table.

Table 6. Analysis of variance (ANOVA) chart for thermoacoustic efficiency at "Junction". Faced centered design, FCD.

\begin{tabular}{cccccc}
\hline Coefficients & SS & Df & MS & F-Value & p-Value \\
\hline FCD Model & 4.4901 & 9 & 0.4989 & 26.0045 & 0.00039 \\
A (f) & 0.3214 & 1 & 0.3214 & 16.7396 & 0.00346 \\
B $\left(D_{C}\right)$ & 1.3072 & 1 & 1.3072 & 68.0833 & 0.000007 \\
C $\left(D_{L}\right)$ & 2.0495 & 1 & 2.0495 & 106.7448 & 0.000002 \\
AB & 0.1345 & 1 & 0.1345 & 7.0052 & 0.01785 \\
AC & 0.2831 & 1 & 0.2831 & 14.7448 & 0.00086 \\
BC & 0.4560 & 1 & 0.4560 & 23.75 & 0.00018 \\
Residual & 0.1151 & 6 & 0.0192 & - & - \\
Lack of fit & 0.1151 & 5 & 0.0230 & - & - \\
Total & 4.6052 & 15 & - & - & - \\
\hline
\end{tabular}

The verification of the quality of the adjustment is satisfactory, since $R^{2}=0.975$ was obtained. This indicates that, within the established range, the DeltaEC simulated results are comparable with the RSM predicted results.

\subsection{Parametric Analysis}

The contour plots, as well as the three-dimensional response surfaces shown in Figures 7-9, are graphic representations of the generated regression model. These plots ease the visualisation of the optimum region proximity. Besides, they describe the interaction between the different parameters studied for thermoacoustic efficiency, evaluated at the "Junction" of the TA-SLiCE prototype.

Figure 7 shows the combined effect of the inner diameters of compliance and inertance on thermoacoustic efficiency. In these plots, the response surface for $\eta_{t a}$ versus the compliance diameter $\left(\mathrm{B}=D_{C}\right)$, and the inertance diameter $\left(C=D_{L}\right)$ is displayed, where the operating frequency of the system is set at its midrange value $(f=40 \mathrm{~Hz})$.

Figure 7 indicates $\eta_{t a}$ reduction with the increase of the inertance inner diameter for a low compliance inner diameter. It means that, for this condition, finer ducts $\left(D_{L}<13 \mathrm{~mm}\right)$ can offer a better thermoacoustic performance. On the contrary, at high compliance diameter, increasing the inertance diameter results in a local improvement of the $\eta_{t a}$, and then it decreases. This phenomenon can be explained by the fact that an inertance to compliance diameter ratio must be maintained to ensure the adequacy of the phase difference between the velocity and the pressure of the acoustic wave. Thus, the feedback branch has to be carefully configured, so that the wave has to leave it with a velocity to pressure phasing close to $0^{\circ}$, a requirement which corresponds to a progressive wave. Setting the 
operating frequency at $40 \mathrm{~Hz}$, the diameter ratio reveals an improvement with a local optimum of $\eta_{t a}=6.496 \%$ for $D_{L}=14.69 \mathrm{~mm}$ and $D_{C}=47.95 \mathrm{~mm}$.

a)

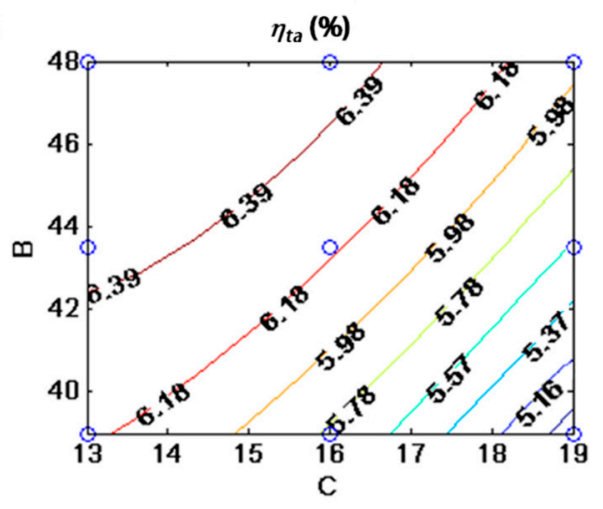

b)

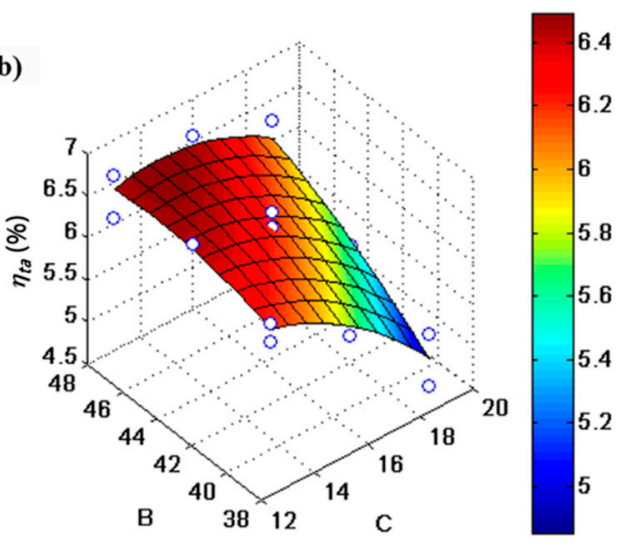

Figure 7. Contour plot (a) and 3D-response surface (b) of thermoacoustic efficiency $\eta_{t a}$ at "Junction", against the combined effect of compliance and inertance inner diameters, B, and C, respectively.

Figure 8 shows the combined effect of the frequency and the inertance inner diameter on the thermoacoustic efficiency. In this graph, the response surface for $\eta_{t a}$ versus the frequency $(A=f)$ and the inertance inner diameter $\left(\mathrm{C}=D_{L}\right)$ is displayed. The compliance inner diameter remains at its mid-range value $\left(D_{C}=43.5 \mathrm{~mm}\right)$. In addition, it shows a $\eta_{t a}$ drop with the increment of the inertance inner diameter. At low inertance diameter $\left(D_{L}=13 \mathrm{~mm}\right)$ the decrease in frequency up to $38.33 \mathrm{~Hz}$ produces an increase in efficiency up to a value of $\eta_{t a}=6.4 \%$. When the frequency is still being reduced below $38.33 \mathrm{~Hz}$, the efficiency does not continue to increase, moreover, it decreases negligibly.

a)

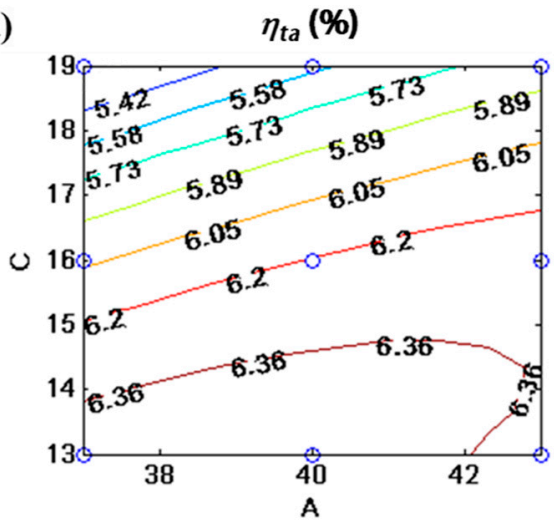

b)

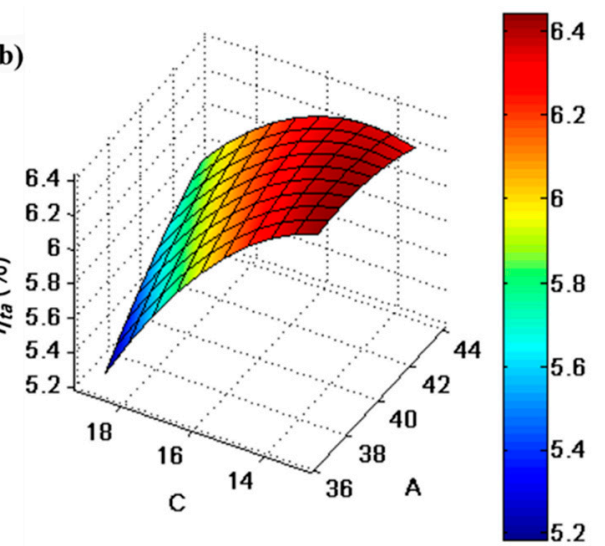

Figure 8. Contour plot (a) and 3D-response surface (b) of thermoacoustic efficiency $\eta_{t a}$ at "Junction", against the combined effect of operating frequency and inertance inner diameter A and C, respectively.

Figure 9 presents the response surface for $\eta_{t a}$ versus the frequency $(A=f)$ and the compliance inner diameter $\left(\mathrm{B}=D_{C}\right)$, keeping the inertance inner diameter constant at $D_{L}=16 \mathrm{~mm}$. At low operating frequency, the $\eta_{t a}$ benefits from the increase of the compliance inner diameter. This is becoming more obvious with the increase of the frequency. Thus, for a fixed $D_{L}=16 \mathrm{~mm}$, the $\mathrm{AB}$ interaction effect reveals a performance improvement with a local optimum of $\eta_{t a}=6.45 \%$ for $f=41 \mathrm{~Hz}, D_{C}=48 \mathrm{~mm}$.

The maximum local efficiency $\eta_{t a}=6.546 \%$ has been obtained by applying RMS for a frequency of $37 \mathrm{~Hz}$ and an inertance and compliance inner diameter of $48.79 \mathrm{~mm}$ and $13.58 \mathrm{~mm}$, respectively. 
a)

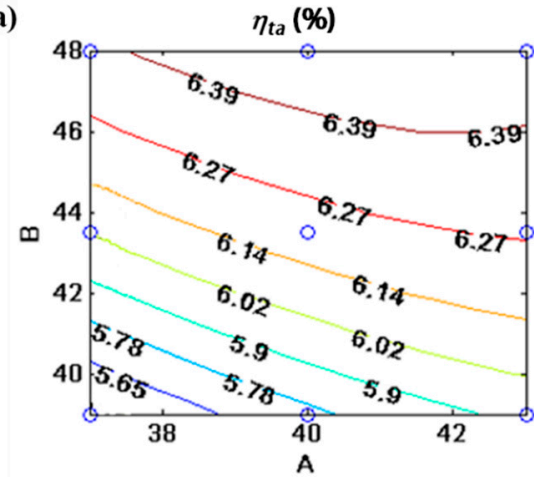

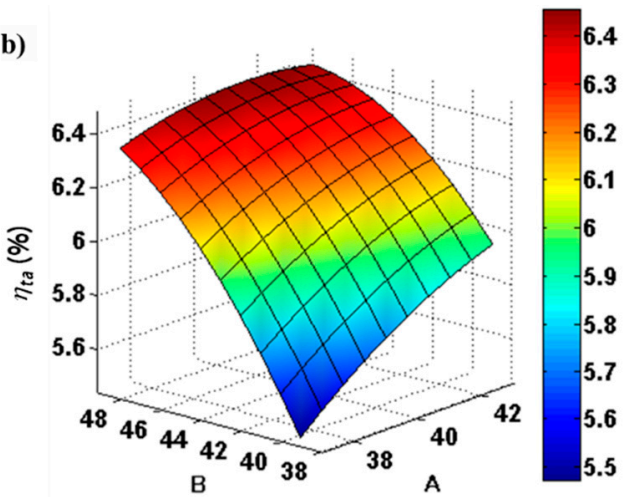

b)

Figure 9. Contour plot (a) and 3D-response surface (b) of thermoacoustic efficiency $\eta_{t a}$ at "Junction", against the combined effect of operating frequency and compliance inner diameter A and B, respectively.

\section{Discussion and Conclusions}

In this paper, a sensitivity study based on DOE has been performed. The conclusions of the present DOE application are restricted to the impact of seven design parameters on the TA-SLiCE performance indicator $\left(y=\eta_{t a}[\%]\right)$. They are thus valid only for the arranged analysis range $(25 \%$ of the extreme range). Given the complexity of the problem, which involves a large number of parameters, a dimensionality reduction has been made applying a "resolution IV" FFD. The resulting linear model has been fitted to the response values for the determination of the significant factors, which proved to be the frequency, the compliance, and the inertance inner diameters.

In addition, a thermoacoustic efficiency optimization of approximately $6 \%$ has been achieved applying RSM. The FCD analysis has provided the optimized combination of the three relevant parameters along with the response surface plots and the contour plots. The predicted results from RSM are largely similar to the values simulated with DeltaEC. Thus, the quadratic model is adequate to evaluate the factor effects and their interactions within the established range. The results obtained confirm the significant influence of the frequency, the compliance and the inertance inner diameters on the performance of the TA-SLiCE studied. The thermoacoustic efficiency is reduced by increasing the inertance inner diameter, while it increases with the increment in the compliance inner diameter. Regarding frequency, the efficiency initially increases from 37 to $41 \mathrm{~Hz}$ and thereafter decreases with increasing frequency. The present study also concludes that interactions, mainly AC and BC, play a modest but significant role on thermoacoustic efficiency. However, the rest of second order and higher order interactions are negligible.

The results obtained infer the possibility of achieving an even greater increase in thermoacoustic efficiency if the range of analysis of the parameters is expanded over $25 \%$. Thus, it is planned to perform a similar study with the expansion of analysis range. Furthermore, a lab prototype of the modeled TA-SLiCE has been successfully built and experimental tests will add into the present work. In addition, extra design parameters will be added to the present analysis as factors in order to extend it to the other two branches of the prototype: the core branch and the power extraction branch. This would increase the knowledge of the TA-SLiCE prototype in terms of performance and functional requirements.

Author Contributions: Conceptualization of the design, C.I. and J.G.; methodology, C.I. and J.L.O.; software, C.I. and J.G.; validation, C.I., J.L.O. and J.V.; formal analysis, C.I. and J.L.O.; investigation, C.I. and J.L.O.; resources, J.L.O. and J.V.; data curation, C.I.; writing — original draft preparation, C.I., J.L.O. and J.V.; writing-review and editing, C.I., J.L.O. and J.V.; visualization, C.I.; supervision, J.L.O. and J.V.; project administration, J.L.O.; funding acquisition, J.V. and J.L.O.

Funding: This research was funded by Comunidad de Madrid, grant number SEGVAUTO 4.0-CM-P2018EEMT-4362 and by the Agencia Estatal de Investigación RETOS 2018-RTI2018-095923-B-C22.

Conflicts of Interest: The authors declare no conflict of interest. 


\section{References}

1. U.S. Department of Energy Energy efficiency \& Renewable Energy. Available online: https://www. fueleconomy.gov/feg/atv.shtml (accessed on 11 October 2019).

2. Kölsch, B.; Radulovic, J. Utilisation of diesel engine waste heat by Organic Rankine Cycle. Appl. Therm. Eng. 2015, 78, 437-448. [CrossRef]

3. Sahoo, D.; Kotrba, A.; Steiner, T.; Swift, G. Waste Heat Recovery for Light-Duty Truck Application Using ThermoAcoustic Converter Technology. SAE Int. J. Engines 2017, 10, 196-202. [CrossRef]

4. Kim, T.Y.; Kwak, J.; Kim, B. Energy harvesting performance of hexagonal shaped thermoelectric generator for passenger vehicle applications: An experimental approach. Energy Convers. Manag. 2018, 160, $14-21$. [CrossRef]

5. Fernández-Yáñez, P.; Armas, O.; Kiwan, R.; Stefanopoulou, A.G.; Boehman, A.L. A thermoelectric generator in exhaust systems of spark-ignition and compression-ignition engines. A comparison with an electric turbo-generator. Appl. Energy 2018, 229, 80-87. [CrossRef]

6. Iniesta, C.; Olazagoitia, J.L.; Vinolas, J.; Aranceta, J. Review of travelling-wave thermoacoustic electric-generator technology. Proc. Inst. Mech. Eng. Part A J. Power Energy 2018, 232, 940-957. [CrossRef]

7. Karlsson, M.; Åbom, M.; Lalit, M.; Glav, R. A Note on the Applicability of Thermo-Acoustic Engines for Automotive Waste Heat Recovery. SAE Int. J. Mater. Manuf. 2016, 9, 286-293. [CrossRef]

8. Swift, G.W. Thermoacoustics: A Unifying Perspective for Some Engines and Refrigerators, 2nd ed.; ASA Press/Springer: Cham, Switzerland, 2017; ISBN 978-3-319-66932-8.

9. Ward, B.; Clark, J.; Swift, G. Design Environment for Low-Amplitude Thermoacoustic Energy Conversion DeltaEC Version 6.4b2; Users Guide; Los Alamos National Laboratory: Los Alamos, NM, USA, 2016.

10. Saechan, P.; Jaworski, A.J. Numerical studies of co-axial travelling-wave thermoacoustic cooler powered by standing-wave thermoacoustic engine. Renew. Energy 2019, 139, 600-610. [CrossRef]

11. Backhaus, S.; Swift, G.W. A thermoacoustic-Stirling heat engine: Detailed study. J. Acoust. Soc. Am. 2000, 107, 3148-3166. [CrossRef] [PubMed]

12. Petach, M.; Tward, E.; Backhaus, S. Design and Testing of A Thermal to Electric Power Converter Based On Thermoacoustic Technology. In Proceedings of the 2nd International Energy Conversion Engineering Conference, American Institute of Aeronautics and Astronauti, Providence, RI, USA, 16-19 August 2004.

13. Yang, P.; Liu, Y.-W.; Zhong, G.-Y. Prediction and parametric analysis of acoustic streaming in a thermoacoustic Stirling heat engine with a jet pump using response surface methodology. Appl. Therm. Eng. 2016, 103, 1004-1013. [CrossRef]

14. Hariharan, N.M.; Sivashanmugam, P.; Kasthurirengan, S. Optimization of thermoacoustic refrigerator using response surface methodology. J. Hydrodyn. 2013, 25, 72-82. [CrossRef]

15. Babu, K.A.; Sherjin, P. Experimental investigations of the performance of a thermoacoustic refrigerator based on the Taguchi method. J. Mech. Sci. Technol. 2018, 32, 929-935. [CrossRef]

16. Desai, A.B.; Desai, K.P.; Naik, H.B.; Atrey, M.D. Optimization of thermoacoustic engine driven thermoacoustic refrigerator using response surface methodology. IOP Conf. Ser. Mater. Sci. Eng. 2017, 171, 012132. [CrossRef]

17. Hariharan, N.M.; Sivashanmugam, P.; Kasthurirengan, S. Optimization of thermoacoustic primemover using response surface methodology. HVAC R Res. 2012, 18, 890-903.

18. Yang, P.; Chen, H.; Liu, Y.W. Application of response surface methodology and desirability approach to investigate and optimize the jet pump in a thermoacoustic Stirling heat engine. Appl. Therm. Eng. 2017, 127, 1005-1014. [CrossRef]

19. Box, G.; Hunter, J.S.; Hunter, W. Statistics Experimenters; Wiley: New York, NY, USA, 2009; pp. 1-655.

20. Ihaka, R.; Gentleman, R. R: A Language for Data Analysis and Graphics. J. Comput. Graph. Stat. 1996, 5, 299-314.

21. Dunn, K.G. Process. Improvement Using Data; McMaster University: Hamilton, ON, Canada, 2010.

22. Andersson, Ö. Experiment! John Wiley \& Sons: New York, NY, USA, 2012; ISBN 9780470688267.

(C) 2019 by the authors. Licensee MDPI, Basel, Switzerland. This article is an open access article distributed under the terms and conditions of the Creative Commons Attribution (CC BY) license (http://creativecommons.org/licenses/by/4.0/). 